\title{
Disturbed B-lymphocytes selection in autoimmune lymphoproliferative syndrome
}

Aleš Janda, $\stackrel{\text { Affl Aff2 }}{\text { Affich }}$

Corresponding Affiliation: Aff1

Klaus Schwarz,, Aff3 Aff4

Mirjam van der Burg,, Aff5

Werner Vach,, Aff6

Hanna Ijspeert, $\stackrel{\text { Aff5 }}{=}$

Myriam Ricarda Lorenz,

Magdeldin Elgizouli,, Aff1

Kathrin Pieper, $\frac{\text { Aff1 }}{\text { }}$

Paul Fisch, $\frac{\text { Aff7 }}{\underline{4}}$

Joachim Hagel, $\stackrel{\text { Aff1 }}{\text { }}$

Raquel Lorenzetti,, Aff1

Maximilian Seidl, $\underline{\text { Affl Aff7 }}$

Joachim Roesler, $\stackrel{\text { Aff8 }}{\text { }}$

Fabian Hauck,, Aff9

Elisabetta Traggiai, $\underline{\text { Aff10 }}$

Carsten Speckmann, $\underline{\text { Aff1 Aff2 }}$

Anne Rensing-Ehl,

Stephan Ehl,, Aff1 Aff2

Hermann Eibel, $\stackrel{\text { Aff1 }}{\text { }}$

Marta Rizzi,

\begin{tabular}{|l|l|l||}
\hline \multicolumn{2}{|c|}{ ArticleInfo } \\
\hline \hline ArticleID & $:$ & 63 \\
\hline \hline ArticleDOI & $:$ & 10.1186/2194-7791-2-S1-A23 \\
\hline \hline ArticleCitationID & $:$ & A23 \\
\hline \hline ArticleSequenceNumber & $:$ & 23 \\
\hline
\end{tabular}




\begin{tabular}{|l|l|l||}
\hline ArticleCategory & $:$ & Meeting abstract \\
\hline \hline ArticleFirstPage & $:$ & 1 \\
\hline \hline ArticleLastPage & $:$ & 3 \\
\hline \hline ArticleHistory & $:$ & $\begin{array}{l}\text { RegistrationDate } \\
\text { OnlineDate }\end{array} \quad: 2015-7-1$ \\
\hline \hline ArticleCopyright & & $\begin{array}{l}\text { Janda et al.2015 } \\
\text { This article is published under license to BioMed Central }\end{array}$ \\
\hline & $:$ & $\begin{array}{l}\text { Ltd. This is an Open Access article distributed under the } \\
\text { terms of the Creative Commons Attribution License } \\
\text { http://creativecommons.org/licenses/by/4.0), which permits } \\
\text { unrestricted use, distribution, and reproduction in any } \\
\text { medium, provided the original work is properly cited. }\end{array}$ \\
\hline \hline
\end{tabular}

Aff1

Centre for Chronic Immunodeficiency, (CCI), University Medical Centre, Freiburg, Germany

Aff2

Centre for Pediatrics and Adolescent Medicine, University Medical Centre, University of Freiburg, Germany

Aff3

Institute for Transfusion Medicine, University of Ulm, Ulm, Germany

Aff4

Institute for Clinical Transfusion Medicine and Immunogenetics Ulm, German Red Cross Blood Service Baden-Württemberg - Hessen, Ulm, Germany

Aff5

Department of Immunology, Erasmus MC, University Medical Center Rotterdam, Rotterdam, The Netherlands

Aff6

Institute of Medical Biometry and Medical Statistics, University Medical Center, Freiburg, Germany

Aff7

Department of Pathology, Molecular Pathology, University Medical Center, University of Freiburg, Germany

Aff8

Department of Pediatrics, University Clinic Carl Gustav Carus, Dresden, Germany

Aff9

Department of Pediatric Hematology, Oncology and Immunology, Dr von Hauner Children's

Hospital, Ludwig-Maximilians-University, Munich, Germany

Aff10

Novartis Institute for Biomedical Research, Basel, Switzerland

Aff1 1

Clinic for Rheumatology and Clinical Immunology, University Medical Centre, University of Freiburg, Germany

Abstracts of the 51st Workshop for Pediatric Research

51st Workshop for Pediatric Research

Göttingen, Germany

16-17 April 2015 
This supplement has not been sponsored.

Meeting abstracts

\section{Meeting abstract}

Autoimmune lymphoproliferative syndrome (ALPS) is characterized by lymphoproliferative disease, autoimmune cytopenias and increased susceptibility to lymphoid malignancies. Central to the pathophysiology of the disease are defects in the FAS signaling leading to impaired lymphocyte homeostasis. Most of the patients harbor heterozygous germline or somatic mutations in FAS. The hallmark of the disease is the impaired FAS-mediated apoptosis of activated $\mathrm{T}$ cells and presence of atypical "double-negative" $\mathrm{T}$ cells

(CD3+TCRalpha/beta+CD4-CD8-). While FAS is essential for deletion of autoreactive B cells in the germinal center in murine models, the role of FAS in human B cell selection and development of autoimmunity in patients carrying FAS mutation is unclear.

We analyzed patients with somatic $F A S$ mutation or germline $F A S$ mutation plus somatic loss-of-heterozygosity allowing to compare the fate of B cells with impaired versus normal FAS signaling within the same individual. We found in the class-switched memory B cells: 1) accumulation of $F A S$ - mutated B cells, 2) a failure to enrich single $\mathrm{V}$ genes and in single V-D, D-J gene combinations of the B cell receptor variable region, 3) increased frequency of variable regions with higher content of positively charged amino acids and longer CDR3 and 4) maintenance of polyreactive specificities. Importantly, FAS-deficient switched memory B cells showed increased rates of somatic hypermutation. Our data uncover a defect in B cell selection in ALPS patients and indicate a role for B cell dysregulation in the pathogenesis of autoimmunity and B-cell lymphoma in ALPS patients. 